\title{
Rosette-Forming Glioneuronal Tumour of the 4th Ventricle in a NF1 Patient
}

\author{
Murad Alturkustani, Lee-Cyn Ang
}

Can. J. Neurol. Sci. 2012; 39: 95-96

We describe a rosette-forming glioneuronal tumour (RGNT) of the 4th ventricle in a 41 year-old man with neurofibromatosis type 1 (NF1). This patient had a family history of NF1 and presented with café-au-lait spots and multiple neurofibromata. At the age of 16 years (1983), he presented with severe headache and the head computed tomogram (CT) scan showed a 4th ventricular mass. The mass was partially resected and the histology was initially reported as a low grade astrocytoma. The patient died at age 41 years.

The smear cytology from the original biopsy 25 years ago, showed a biphasic lesion composed of piloid cells with elongated fibrillary processes accompanied by Rosenthal fibers, and small round cells with finely granular nuclear chromatin forming rare neurocytic rosette (Figure 1a, b). The paraffin sections revealed a tumour with small round nuclei and scanty cytoplasm forming perivascular pseudorosettes surrounded by a low cellular glial component with piloid cells, microcystic areas and Rosenthal fibers. The perivascular cells were immunoreactive to synaptophysin, microtubule-associated protein 2 (MAP2) and neuron specific enolase (NSE) (Figure 1c-f) and immuno-negative for glial fibrillary acidic protein (GFAP). The glial areas were GFAP immuno-positive. There was no mitotic activity, vascular endothelial proliferation or tumour necrosis.

At the follow-up after 23 years (2006), the patient had spastic gait and the MRI showed a 7 to $8 \mathrm{~mm}$ focus of increased signal in the fourth ventricular region within the periaqueductal grey matter of the right rostral pons (Figure 2a). It had no mass effect or enhancement. He suffered from schizophrenia and depression and two years later (2008), committed suicide. The general autopsy demonstrated findings of NF1 (multiple café-au- lait spots and neurofibromata) and the brain was referred for neuropathological examination.

Neuropathological examination revealed the residual tumour at the rostral pons around the fourth ventricle. The glial component was predominant with numerous microcystic areas. A few microcysts contained clusters of cells with small round nuclei and visible nucleoli. Morphologically, these cells resembled the neurocytic component of the original biopsy, but the neuronal markers were immuno-negative. Although relatively well circumscribed, there were atypical glial cells in the pontine tegmentum (Figure $2 \mathrm{~b}-\mathrm{d}$ ). In the frontal cortex and

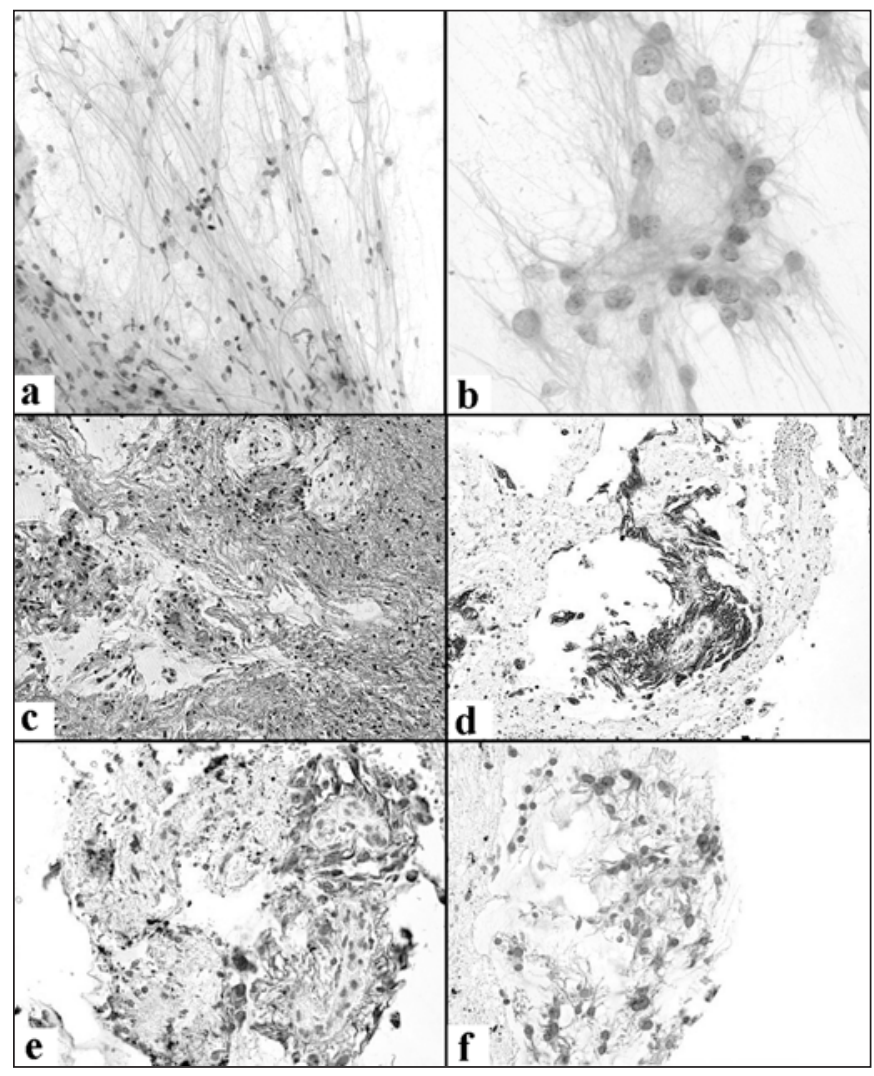

Figure 1: Smear of the surgical biopsy shows the two cytological components of the lesion: a) Glial component consists of piloid cells accompanied by Rosenthal fibers (H\&E x200), b) Neuronal component in form of neurocytic rosettes (H\&E x400). Surgical biopsy shows: c) Neuronal component mainly presents as perivascular pseudorosettes (H\&E x200). These cells are positive to different neuronal markers: d) (MAP2 x200), e) (NSE x400) and f) (Synaptophysin x400).

left amygdala, multiple microhamartomas consisting of mixture of clustered oligodendrocyte-like cells and small ganglion-like cells were present (Figure 2e). There were frequent plaques with amyloid core in the temporal and cingulate cortices. These were

From the Department of Pathology (Neuropathology) (LCA, MA), University Hospital, University of Western Ontario, London, Ontario, Canada; Department of Pathology (MA), King Abdulaziz University and Hospital, Jeddah, Kingdom of Saudi Arabia.

Received June 6, 2011. Final Revisions Submitted Aigist 29, 2011.

Correspondence to: Murad Alturkustani, University Hospital, University of Western Ontario, 339 Windermere Rd, London, Ontario, N6A5A5, Canada. 


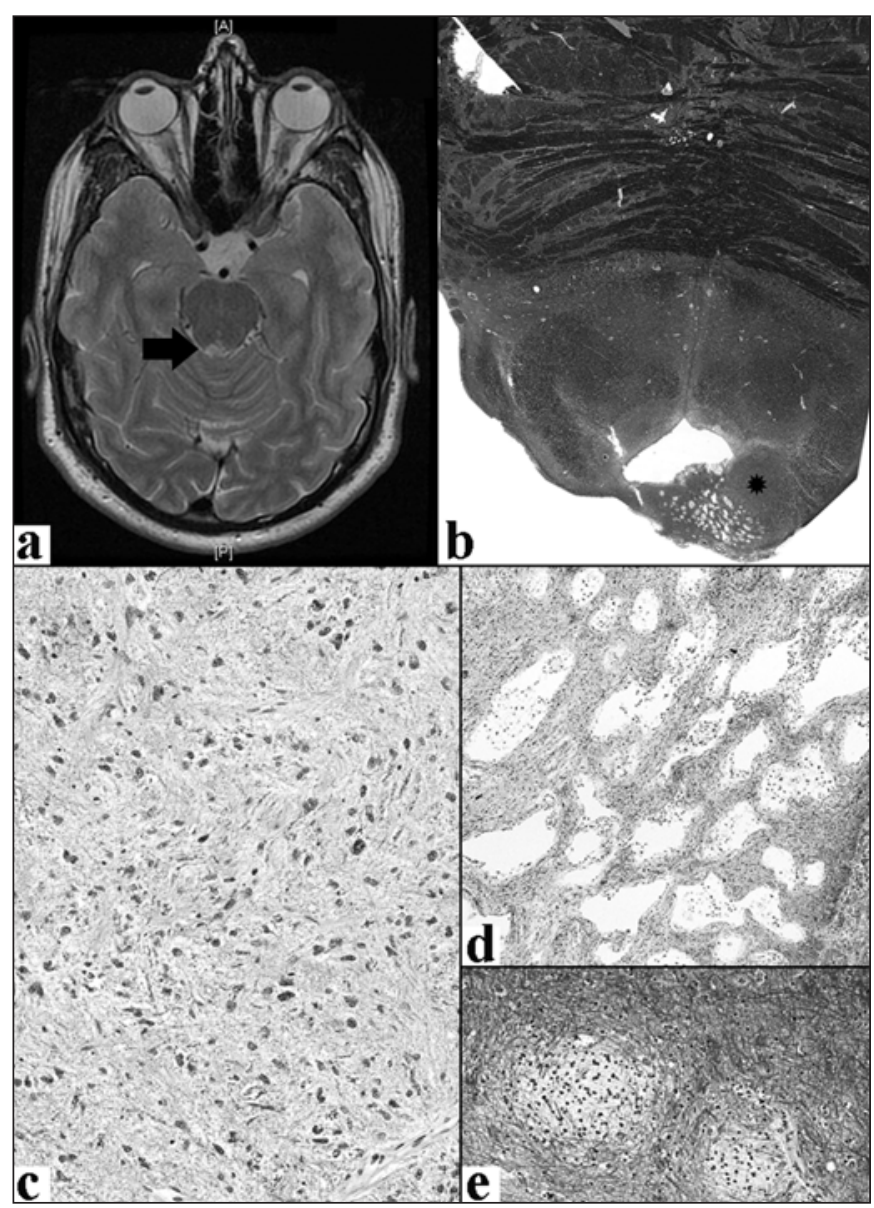

Figure 2: a) T2 weighted MRI without gadolinium shows a 7 to $8 \mathrm{~mm}$ focus of increased signal in 4th ventricular region (arrow). b) Horizontal section across the rostral pons shows the well circumscribed nodule (asterisk), (H\&E/Luxol fast blue). c) Atypical glial cells in the pontine tegmentum in the adjacent brain stem (H\&E/Luxol fast blue x200), d) The astrocytic component is dominant with numerous microcystic areas (H\&E/Luxol fast blue x40), e) Microhamartoma (H\&E/Luxol fast blue $x 100)$.

highlighted by the Bielschowsky and Congo red stains as well as by amyloid precursor protein and beta-amyloid immunohistochemistry. However, no neuritic plaques or tau positive neurofibrillary tangles were seen.

Rosette-forming glioneuronal tumour of the fourth ventricle, which is included as a grade I tumour in the recent World Health Organization classification of central nervous system tumours ${ }^{1}$, was first described as a cerebellar form of dysembryoplastic neuroepithelial tumour in $1995^{2}$. Subsequently, Komori et al introduced the term RGNT of the fourth ventricle in their description of 11 patients in $2002^{3}$. In the English literature, there are 47 cases reported so far ${ }^{4}$. These cases showed tumour predilection for young adults with the mean age of about 30 years and female predominance (31 Female (F), 16 Male (M)). Most of the symptomatic cases presented with clinical features of increased intracranial pressure (i.e. headache, nausea, vomiting). Ataxia, vertigo, and cranial nerve palsies had been reported occasionally, and rarely seizures, nystagmus and hyperacusis as well. The MRIs in most cases showed solid and cystic lesions around the fourth ventricle, which were isointense or hypointense on T1-weighted images and hyperintense on T2weighted images with variable enhancement.

This tumour is characterized by the presence of the biphasic components (neuronal and glial). The glial portion of this tumour for the majority ( 25 out of 47 reported cases) resembles pilocytic astrocytoma (PA) including: piloid cells with hair-like cytoplasmic processes, microcytic matrix, Rosenthal fibers, eosinophilic granular bodies, and microcalcification. The neuronal component consists of uniform neurocytes forming rosettes and/or perivascular pseudorosettes. Anaplastic features such as mitoses, necrosis or endothelial proliferation have not been reported ${ }^{1}$. The literature revealed no evidence of clinical recurrence except for two cases treated with post-surgical radiation, despite the fact that about half of these tumours have not been completely resected.

Rosette-forming glioneuronal tumour has only been reported once previously in association with NF1, however that particular tumour was located in the chiasmal optic nerve and not the fourth ventricular region ${ }^{5}$. Apart from its unusual location, this tumour showed the typical histological features of RGNT. Since the association with NF1 is documented in two (including our case) of the $47(4.25 \%)$ reported cases, we believe this may not be purely coincidental. Although the tumour in our patient followed a benign clinical course of 25 years, the microscopic findings based on autopsy material showed the persistence of an infiltrative glial component consistent with its neoplastic nature. In spite of its indolent nature, this tumor bore no resemblance to previously described hamartoma or developmental anomalies in the same region ${ }^{6}$.

As far as we know, this is the first documented case in the English literature of a RGNT located at the fourth ventricular region in a NF1 patient.

\section{REFERENCES}

1. Hainfellner JA, Scheithauer BW, Giangaspero F, Rosenblum MK. Rosette-forming glioneuronal tumour of the fourth ventricle. In: Louis DN, Ohgaki H, Wiestler OD, Cavenee WK, editors. WHO Classification of Tumours of the Central Nervous System, 4th ed. IARC, Lyon. 2007; 115-6.

2. Kuchelmeister K, Demirel T, Schlörer E, Bergmann M, Gullotta F. Dysembryoplastic neuroepithelial tumour of the cerebellum. Acta Neuropathol. 1995; 89: 385-90.

3. Komori T, Scheithauer BW, Hirose T. A rosette-forming glioneuronal tumor of the fourth ventricle: infratentorial form of dysembryoplastic neuroepithelial tumor? Am J Surg Pathol. 2002; 26: 582-91.

4. Solis O, Mehta R, Lai A, et al. Rosette-forming glioneuronal tumor: a pineal region case with IDH1 and IDH2 mutation analyses and literature review of 43 cases. J Neurooncol. 2011; 102 (3): 477-84.

5. Scheithauer BW, Silva AI, Ketterling RP, Pula J, Lininger JF, Krinock MJ. Rosette-forming glioneuronal tumor: report of a chiasmal-optic nerve example in neurofibromatosis type 1: special pathology report. Neurosurgery. 2009; 64: E771-2.

6. Delalande O, Rodriguez D, Chiron C, Fohlen M. Successful surgical relief of seizures associated with hamartoma of the floor of the fourth ventricle in children: report of two cases. Neurosurgery 2001; 49: 726-31. 